\title{
The Enhancing Secondary Prevention in Coronary Artery Disease trial
}

\author{
Finlay A. McAlister MD MSc, Miriam Fradette BSc Pharm, Sumit R. Majumdar MD MPH, \\ Randall Williams MD, Michelle Graham MD, James McMeekin MD, William A. Ghali MD MPH, \\ Ross T. Tsuyuki PharmD MSc, Merril L. Knudtson MD, Jeremy Grimshaw MB ChB PhD
}

Previously published at www.cmaj.ca

\section{ABSTRACT}

Background: Proven efficacious therapies are sometimes underused in patients with chronic cardiac conditions, resulting in suboptimal outcomes. We evaluated whether evidence summaries, which were either unsigned or signed by local opinion leaders, improved the quality of secondary prevention care delivered by primary care physicians of patients with coronary artery disease.

Methods: We performed a randomized trial, clustered at the level of the primary care physician, with 3 study arms: control, unsigned statements or opinion leader statements. The statements were faxed to primary care physicians of adults with coronary artery disease at the time of elective cardiac catheterization. The primary outcome was improvement in statin management (initiation or dose increase) 6 months after catheterization.

Results: We enrolled 480 adults from 252 practices. Although statin use was high at baseline $(n=316[66 \%])$, most patients were taking a low dose (mean $32 \%$ of the guideline-recommended dose), and their low-density lipoprotein (LDL) cholesterol levels were elevated (mean $3.09 \mathrm{mmol} / \mathrm{L}$ ). Six months after catheterization, statin management had improved in 79 of 157 patients $(50 \%)$ in the control arm, 85 of $158(54 \%)$ patients in the unsigned statement group (adjusted odds ratio [OR] 1.18, 95\% Cl 0.71-1.94, $p=0.52$ ) and 99 of 165 $(60 \%)$ patients in the opinion leader statement group (adjusted OR 1.51, 95\% Cl 0.94-2.42, $p=0.09$ ). The mean fasting LDL cholesterol levels after 6 months were similar in all 3 study arms: 2.35 (standard deviation [SD] 0.86) $\mathrm{mmol} / \mathrm{L}$ in the control arm compared with 2.24 (SD 0.73) among those in the opinion leader group $(p=0.48)$ and 2.19 (SD $0.68)$ in the unsigned statement group $(p=0.32)$.

Interpretation: Faxed evidence reminders for primary care physicians, even when endorsed by local opinion leaders, were insufficient to optimize the quality of care for adults with coronary artery disease. ClinicalTrials.gov trial register no. NCT00175240.

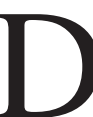
espite the abundant evidence base for the secondary prevention of coronary artery disease, ${ }^{1}$ many of these therapies are underused in clinical practice. ${ }^{1-4}$ These gaps between evidence and clinical reality are linked to poor outcomes for patients. ${ }^{5}$ Improved uptake of secondary-prevention therapies would reduce cardiac mor- bidity and mortality. ${ }^{6}$ However, most quality-improvement initiatives in coronary artery disease have focused on patients in hospital. Few studies have evaluated means of translating evidence into clinical practice for outpatients cared for by primary care physicians.

Previously, ${ }^{8}$ we developed and tested the Local Opinion Leader Statement (Appendix 1, available at www.cmaj.ca /cgi/content/full/cmaj.090917/DC1), a quality-improvement tool consisting of a 1-page summary of evidence with explicit treatment advice about secondary prevention of coronary artery disease. This summary was endorsed by local opinion leaders and was faxed to the primary care physicians of patients with coronary artery disease. Although this fax did not lead to a significant improvement in statin prescribing, our pilot trial was small (117 patients) and enrolled patients with chronic coronary artery disease at the time they presented to their community pharmacy for medication refills. We hypothesized that this was not a "teachable moment" and that if the intervention was given at a time when the diagnosis was made, it would be more influential on the primary care physician.

Thus, we designed this trial to test the impact of the opinion leader statement if it was sent to the primary physician at the time when patients were diagnosed with coronary artery disease. In addition, because local opinion leaders are not always selfevident and conducting surveys to identify them for each condition and in each locale would be time-consuming and expensive, we also evaluated the impact of an unsigned statement.

\section{Methods}

The protocol for the Enhancing Secondary Prevention in Coronary Artery Disease (ESP-CAD) trial, including details about the primary care physician survey that we conducted to identify local opinion leaders for coronary artery disease in

\footnotetext{
From the Department of Medicine (McAlister, Majumdar, Graham, Tsuyuki) University of Alberta; the Epidemiology Coordinating and Research Centre (McAlister, Fradette, Majumdar, Graham, Tsuyuki), University of Alberta; the Mazankowski Alberta Heart Institute (McAlister, Graham, Tsuyuki), University of Alberta; the Royal Alexandra Hospital (Williams), Edmonton, Alta.; the Department of Medicine (McMeekin, Ghali, Knudtson), University of Calgary, Calgary, Alta.; and the University of Ottawa Health Research Unit (Grimshaw), Ottawa, Ont.
}

Cite as CMAJ 2009. DOI:10.1503/cmaj.090917 
Edmonton and Calgary, has been published. ${ }^{1}$ In brief, we conducted a randomized clinical trial in these cities clustered at the level of the primary care physician to avoid contamination. We tested 3 interventions: usual care, opinion leader statements and unsigned statements (Figure 1). Patients were screened in all 3 cardiac catheterization laboratories in Alberta (total population about 3.3 million people) between June 2005 and January 2008 to identify adult patients with coronary artery disease $(\geq 50 \%$ stenosis in at least 1 coronary vessel) detected by elective cardiac catheterization. We included patients who were eligible for but not already taking a statin or who were on a suboptimal regimen (e.g., taking a low-dose statin but whose low-density lipoprotein [LDL] cholesterol levels remained elevated) (Table 1). The exclusion criteria are listed in Figure 1.
Randomization (1:1:1) was performed after completion of the patient's angiogram. Patients were randomly assigned to groups by use of a computer-generated central randomization system with concealment of the randomization list. Although the primary care physicians were not blinded to the allocation status, both allocation concealment and blinding were achieved for investigators, patients, outcome assessors and analysts.

The institutional ethics review boards at the University of Alberta and the University of Calgary approved this study, and all patients provided written informed consent.

\section{Interventions}

The opinion leader statements were imprinted with the name of the participating patient, addressed directly to the patient's

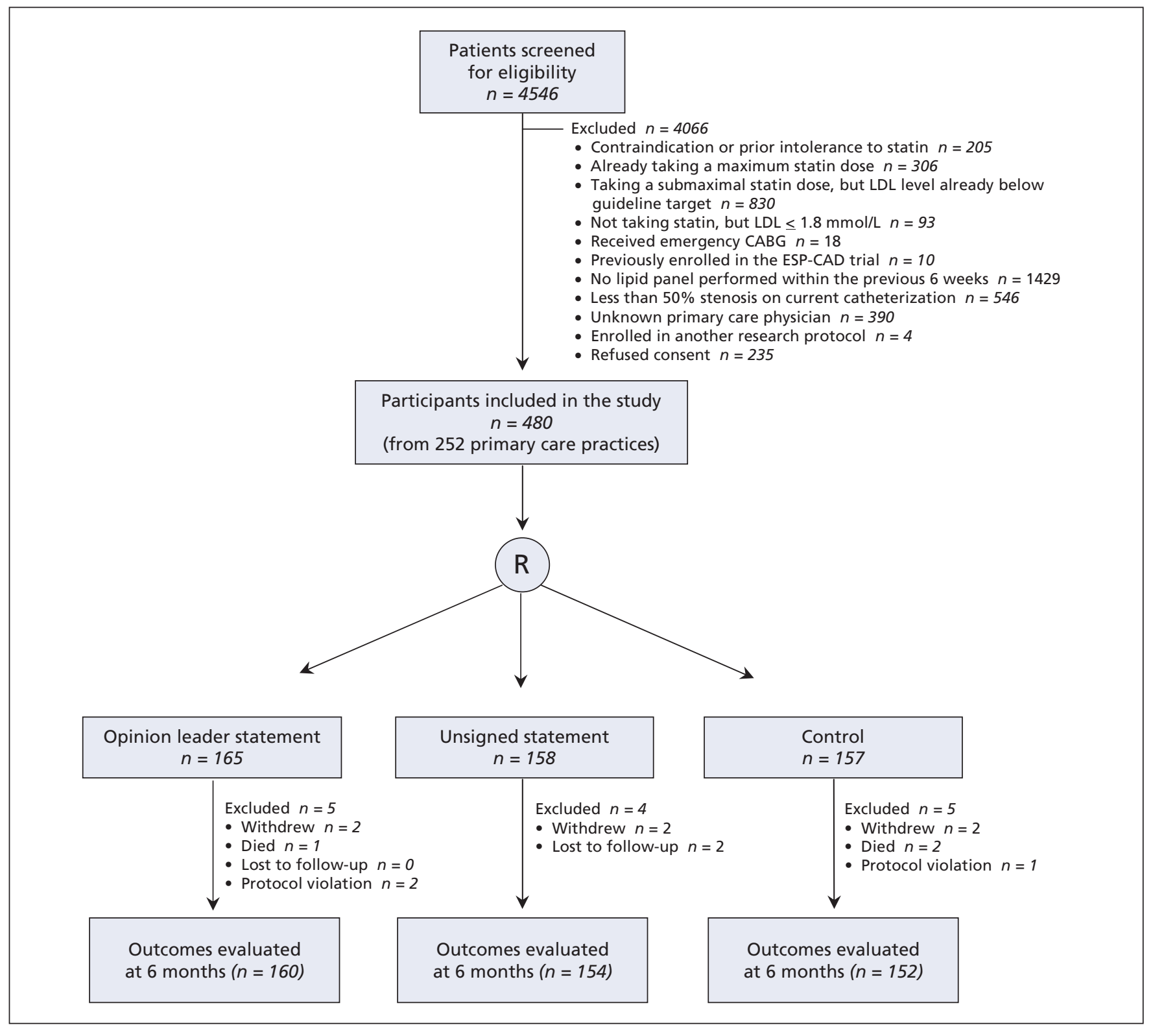

Figure 1: Selection and randomization of patients included in the Enhancing Secondary Prevention in Coronary Artery Disease (ESP-CAD) trial. $L D L=$ low-density lipoprotein, $C A B G=$ coronary artery bypass graft. 
Table 1: Characteristics at the time of cardiac catheterization of patients included in the Enhancing Secondary Prevention in Coronary Artery Disease trial

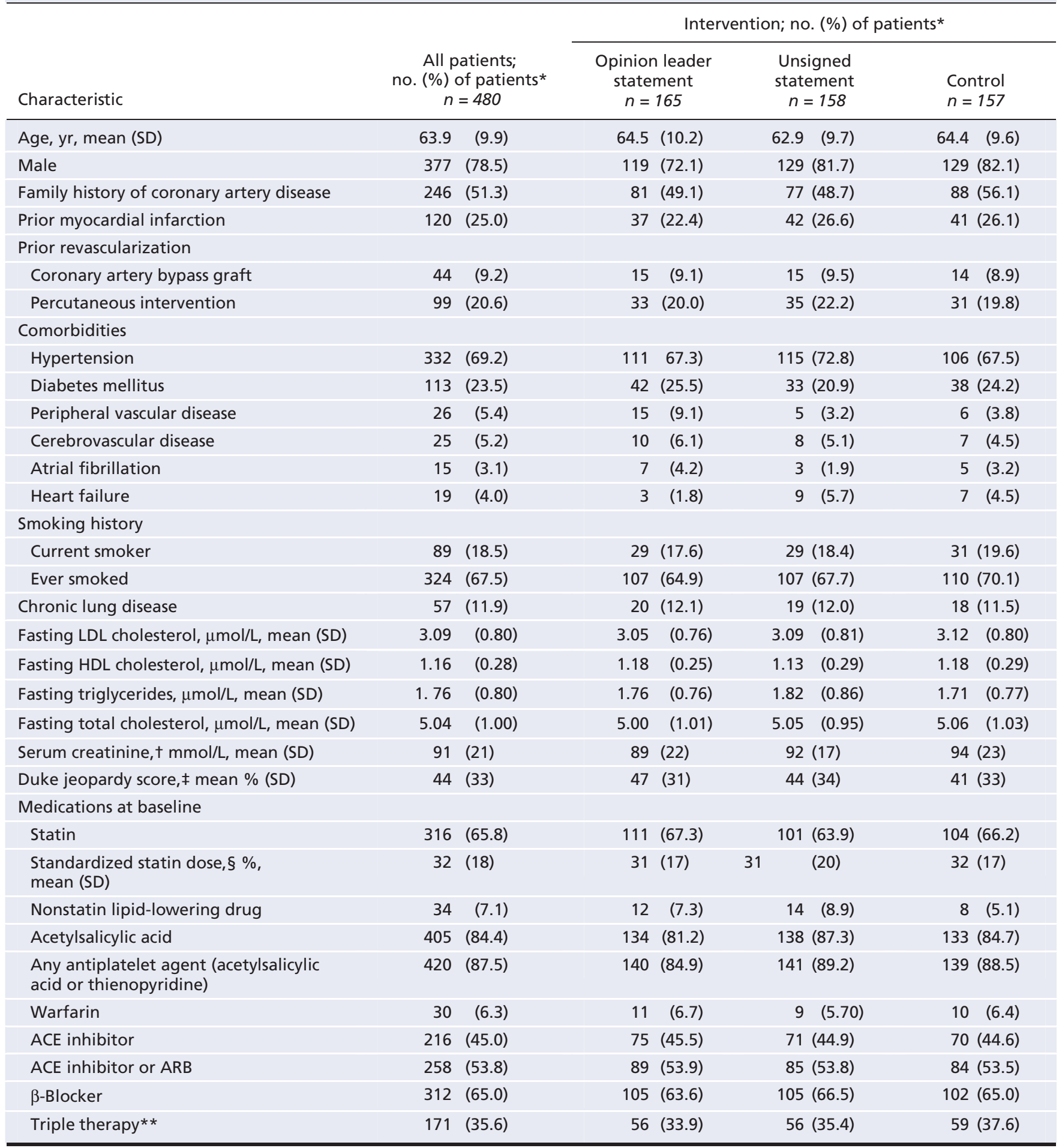

Note: $\mathrm{ACE}=$ angiotensin-converting enzyme, $\mathrm{ARB}=$ angiotensin-receptor blocker, $\mathrm{LDL}=$ low-density lipoprotein, $\mathrm{HDL}=$ high-density lipoprotein, $\mathrm{SD}=\mathrm{standard}$ deviation.

*Unless stated otherwise.

tSerum creatinine data were available only for 274 patients ( $n=89,86,99$ in the opinion leader, unsigned statement and control groups, respectively).

$\neq$ A measure of the proportion of the myocardium at risk from ischemia.

$\S$ Calculated for each statin individually as a percentage of the recommended dose (Canadian Cardiovascular Society guideline) and averaged across all patients taking statins.

**Statin, an antiplatelet agent and an ACE inhibitor. 
physician, signed by the local opinion leaders for that city, and faxed automatically by a software program that was developed by the Alberta Provincial Project for Outcome Assessment in Coronary Heart Disease (APPROACH) for this trial. Each physician received a fax containing objective evidence of the patient's coronary artery disease (in the form of a coronary artery diagram $)^{9}$ and either a signed or unsigned statement. These faxes were sent to physicians within a few days of the angiogram. The unsigned statements were identical to the opinion leader statements in content and form but did not contain the opinion leaders' signatures. The unsigned statements were faxed to physicians in the same manner as the opinion leader statements.

\section{Controls}

Physicians of the control patients received a fax containing the coronary artery diagram for their patient (this represents an enhancement over and above usual care in our province). This ensured that the physicians of all participants received the same number of faxes, with the only difference being the content.

\section{Outcomes}

The primary outcome was improved statin management, which we defined as initiation or increased dosage of a statin within the first 6 months after cardiac catheterization. To compare dosing across statins, we calculated the mean standardized statin dose for each prescribed statin as a percentage of the target dose specified for each agent in the guidelines. ${ }^{10}$ We also collected data about a number of secondary outcomes (Table 2). Medication outcomes were based on patient self-report (with cross-referencing to pharmacy records), and laboratory data and clinical outcomes were extracted from medical records. Follow-up data were collected by independent assessors blinded to the groups. The clinical events were

Table 2: Outcomes 6 months after cardiac catheterization

\begin{tabular}{|c|c|c|c|c|c|c|}
\hline \multirow{3}{*}{$\begin{array}{l}\text { Outcome } \\
\text { Statin initiation or increase } \\
\text { in dose }\end{array}$} & \multicolumn{3}{|c|}{ Intervention; no. (\%) of people* } & \multicolumn{3}{|c|}{$p$ valuet } \\
\hline & $\begin{array}{l}\text { Opinion leader } \\
\text { statement } \\
n=165\end{array}$ & \multirow{2}{*}{$\begin{array}{c}\begin{array}{c}\text { Unsigned } \\
\text { statement } \\
n=158\end{array} \\
85(54.0)\end{array}$} & $\begin{array}{l}\text { Control } \\
n=157\end{array}$ & \multirow{2}{*}{$\begin{array}{c}\text { Opinion leader } \\
\text { statement } \\
\text { v. control }\end{array}$} & \multirow{2}{*}{$\begin{array}{c}\begin{array}{c}\text { Unsigned } \\
\text { statement } \\
\text { v. control }\end{array} \\
0.52\end{array}$} & \multirow{2}{*}{$\begin{array}{c}\begin{array}{c}\text { Either } \\
\text { statement } \\
\text { v. control }\end{array} \\
0.17\end{array}$} \\
\hline & $99(60.0)$ & & $79(50.0)$ & & & \\
\hline Taking a statin & 138 (83.6) & $139(88.0)$ & $129(82.2)$ & 0.99 & 0.43 & 0.62 \\
\hline $\begin{array}{l}\text { Standardized statin dose, mean } \\
\text { (SD) } \ddagger\end{array}$ & $43(25)$ & $42(26)$ & $42(26)$ & 0.82 & 0.91 & 1.0 \\
\hline Taking another lipid-lowering drug & $20(12.1)$ & $22(13.9)$ & $19(12.1)$ & 0.99 & 0.63 & 0.78 \\
\hline Acetylsalicylic acid & $140(84.8)$ & $136(86.1)$ & $135(86.0)$ & 0.72 & 0.90 & 0.78 \\
\hline Acetylsalicylic acid or thienopyridine & $146(88.5)$ & $138(87.3)$ & 141 (89.8) & 0.64 & 0.32 & 0.40 \\
\hline ACE inhibitor & $90(54.6)$ & $74(46.8)$ & $79(50.3)$ & 0.52 & 0.58 & 0.94 \\
\hline ACE inhibitor or ARB & $107(64.8)$ & $94(59.5)$ & $97 \quad(61.8)$ & 0.70 & 0.87 & 0.90 \\
\hline$\beta$-Blocker & $126(76.4)$ & $126(79.8)$ & $108(68.8)$ & 0.08 & 0.03 & 0.03 \\
\hline Triple therapy§ & $91(55.2)$ & $85(53.8)$ & $86(54.8)$ & 0.93 & 0.94 & 0.93 \\
\hline Stopped smoking** & 11 (37.9) & $9(31.0)$ & $12(38.7)$ & 0.94 & 0.51 & 0.68 \\
\hline $\begin{array}{l}\text { Received smoking cessation advice } \\
\text { or were prescribed a smoking } \\
\text { cessation product** }\end{array}$ & $13(44.8)$ & 11 (37.9) & $17(54.8)$ & 0.45 & 0.20 & 0.24 \\
\hline Deaths & $1 \quad(0.61)$ & (0) & $2 \quad(1.27)$ & 0.62 & 0.25 & 0.25 \\
\hline \multicolumn{7}{|l|}{ No. of patients visiting the ED } \\
\hline Total & $31(18.8)$ & $36(22.8)$ & 25 (15.9) & 0.43 & 0.14 & 0.24 \\
\hline For an atherosclerotic event†† & $2 \quad(6.4)$ & (0) & $1 \quad(4.0)$ & 1.0 & 0.41 & 0.98 \\
\hline \multicolumn{7}{|l|}{$\begin{array}{l}\text { No. of patients admitted to } \\
\text { hospital }\end{array}$} \\
\hline Total & $54 \quad(32.7)$ & $53(33.5)$ & $55(35.0)$ & 0.61 & 0.69 & 0.60 \\
\hline For an atherosclerotic event†† & $3 \quad(5.6)$ & $4 \quad(7.6)$ & $4 \quad(7.3)$ & 0.71 & 0.94 & 0.85 \\
\hline
\end{tabular}


independently adjudicated by 2 blinded investigators (F.A.M. and S.R.M.) who then met to resolve discrepancies.

\section{Statistical analysis}

Statistical analyses were conducted by a statistician blinded to allocation status. Intention-to-treat analyses were carried out with patients as the unit of analysis and causal inference. We used the $\chi^{2}$ test and subsequently generalized estimating equations to adjust for baseline covariables and clustering ${ }^{11}$ to compare the attainment of our primary outcome within 6 months among patients in the control group versus those in the opinion leader statement group, the unsigned statement group and both the opinion leader and unsigned statement groups (because both are point-of-care evidence reminders). We also performed a limited number of posthoc exploratory subgroup analyses defined by the use of statins at baseline, the level of LDL at baseline and the involvement of a specialist after cardiac catheterization. Neither the ESP-CAD study protocol nor the faxed statements advocated for or against specialist involvement after catheterization, and the decision to involve a specialist was left to the discretion of the primary care physician.

We targeted a total sample size of 480 patients (160 patients per arm) to detect a $15 \%$ absolute difference in the primary outcome over control rates, with an $\alpha$ of 0.05 (2-sided) and $80 \%$ power.

\section{Results}

\section{Patient characteristics}

Of the 4546 patients screened, 715 were eligible, and 480 $(67 \%)$ agreed to participate (Figure 1). These patients were drawn from 252 primary care practices. The average cluster size was 1.9 patients per practice and the intraclass correlation coefficient for statin prescribing at 6 months was 0.019 . We evaluated the status of 466 patients $(97 \%)$ after 6 months ( 2 patients were lost to follow-up, 6 withdrew consent, 3 died and 3 were excluded because of protocol violations [ 2 were ineligible at randomization and 1 underwent a lung transplant during follow-up]).

At baseline, there were no statistically significant differences between the groups (Table 1). Although $66 \%$ of patients were taking a statin at baseline, the dosing was relatively low (the mean standardized dose was $32 \%$ of the guideline-recommended therapeutic dose) with no appreciable differences between the treatment arms ( $p=0.87$ for statin use; $p=0.84$ for statin dosing).

\section{Improvement in statin therapy}

At 6 months, statin therapy had improved in all 3 study arms (Table 2) with no statistically significant differences between the arms. Statin therapy had either been initiated or the dose increased in 79 of 157 (50\%) control patients compared with 85 of $158(54 \%)$ patients in the unsigned statement group (adjusted OR 1.18, 95\% CI 0.71-1.94, $p=0.52$ ) and 99 of $165(60 \%)$ patients in the opinion leader statement group (adjusted OR 1.51, 95\% CI 0.94-2.42, $p=0.09$ ).

Improvements were larger among patients who were not taking a statin at baseline (129/164 [79\%] started a statin compared to $134 / 316$ [42\%] taking a statin who had their dose increased) or who had higher baseline LDL cholesterol levels (Figure 2). Although both interventions led to more marked improvements in each subgroup compared with con-

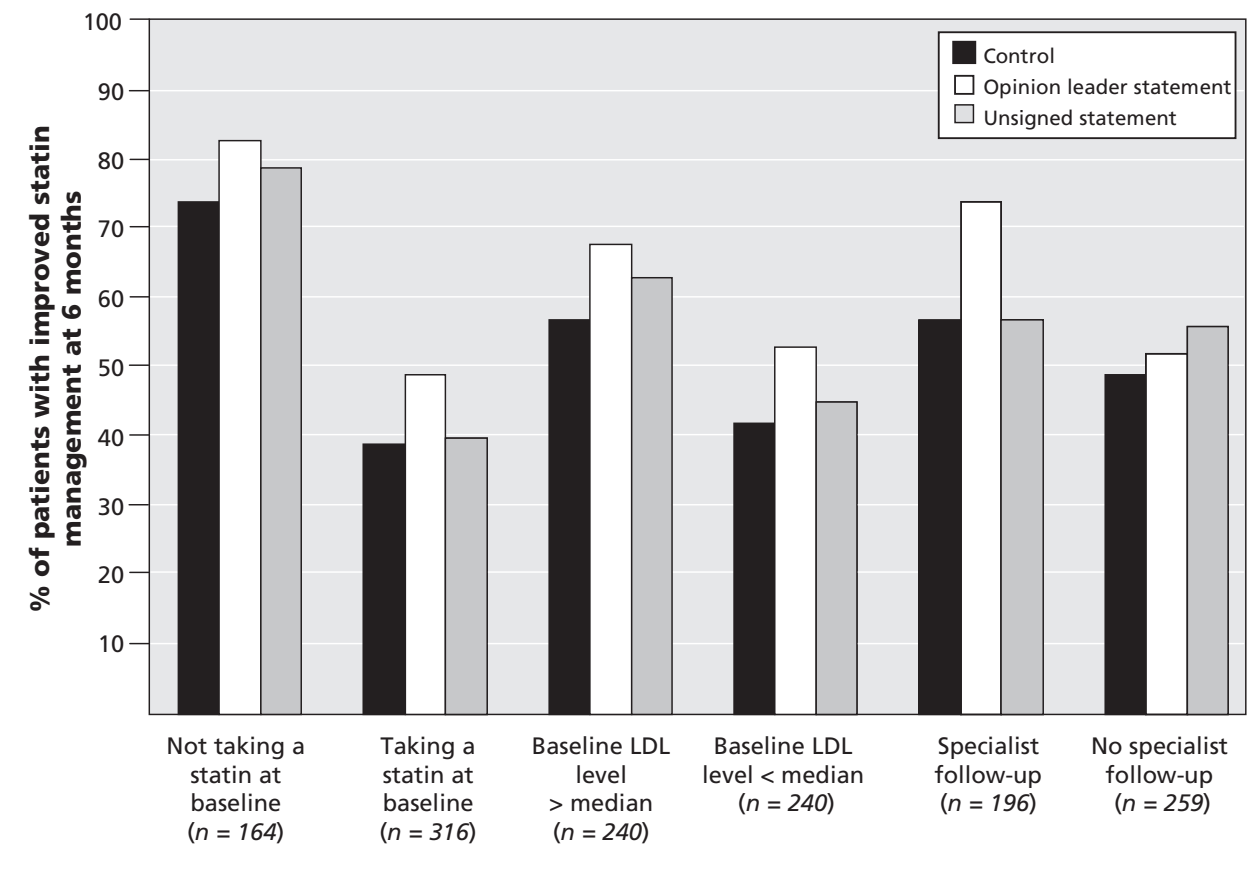

Figure 2: Improvement in statin management (initiation of a statin or doseage increase) 6 months after cardiac catheterization in subgroups. The median low-density lipoprotein (LDL) cholesterol level at baseline was $2.9 \mathrm{mmol} / \mathrm{L}$. 
trol, none of the comparisons were statistically significant (Figure 2). However, the opinion leader statement was associated with a statistically significant improvement over control in the group of patients who were followed by both a specialist and a primary care physician $(74 \%$ v. $57 \%, p=0.04$, adjusted OR 2.41, 95\% CI 1.12-5.18, $p=0.02$ ).

In the multivariable analysis, only elevated LDL cholesterol at baseline (adjusted OR 1.86, 95\% CI 1.29-2.69, $p=0.001$, if baseline LDL was greater than median) and seeing a specialist during follow-up (adjusted OR 1.56, 95\% CI $1.05-2.33, p=0.03$ ) were significantly associated with improved statin therapy. About $40 \%(n=196)$ of the patients were seen by a specialist after cardiac catheterization with no appreciable difference among the groups (73/165 patients in the opinion leader statement arm, 63/158 in the unsigned statement arm, and 60/157 in the control arm; $p=0.52$ ).

\section{Secondary outcomes}

Although we observed improvements in all of the secondary outcomes, none except the use of $\beta$-blockers differed significantly among the trial arms (Table 2). The mean fasting LDL cholesterol levels after 6 months were similar in all 3 study arms: 2.35 (standard deviation [SD] 0.86) $\mathrm{mmol} / \mathrm{L}$ in the control arm compared with 2.24 (SD 0.73) $\mathrm{mmol} / \mathrm{L}$ among those randomized to the opinion leader statement $(p=0.48)$ and 2.19 (SD 0.68) $\mathrm{mmol} / \mathrm{L}$ among patients in the unsigned statement group $(p=0.32)$. However, less than half of the study participants had fasting LDL cholesterol levels of $2.0 \mathrm{mmol} / \mathrm{L}$ or less at 6 months $(74 / 165$ [45\%] in the opinion leader statement group; 60/157 [38\%] in the control group; 55/158 [35\%] in the unsigned statement group). Just over half were taking all 3 recommended secondary-prevention therapies (a statin, an antiplatelet agent and an ACE inhibitor; 91/165 [55\%] in the opinion leader group, 86/157 [55\%] in the control group, and $85 / 158$ [54\%] in the unsigned statement group).

\section{Interpretation}

We observed substantial improvements in the quality of secondary-prevention care for patients with coronary artery disease within 6 months of elective diagnostic cardiac catheterization. Although both of our interventions led to marked improvements over control, neither achieved statistical significance, given the $50 \%$ absolute improvement in the control group.

The magnitude of improvements after cardiac catheterization in all groups was several fold larger than usually seen with quality-improvement strategies. ${ }^{7}$ Thus, our results reinforce the importance of testing quality-improvement interventions in randomized trials ${ }^{12}$ — an old message, but one that is sometimes forgotten in the quality-improvement literature. ${ }^{13}$ Had we conducted our study using the popular before-after design, we would have erroneously concluded that both the opinion leader and unsigned statement were highly effective.

Despite the improvements in statin management that we observed in all 3 arms of our trial, there is still room for improvement. Six months after cardiac catheterization, only half of the participants were taking triple therapy (statin, an antiplatelet agent and an angiotensin-converting-enzyme inhibitor), and, although over $80 \%$ were taking statins, their dosages were still low relative to the doses in clinical trials. ${ }^{10,14}$ As a result, less than half of the participants had attained the guideline-recommended LDL targets. Thus, our inability to show a statistically significant benefit over control cannot merely be attributed to a ceiling effect.

Why were our interventions not more effective? Both the opinion leader and unsigned statements functioned as pointof-care reminders for physicians and mentioned a specific patient, contained explicit treatment recommendations and provided the underlying evidence. Although this strategy should theoretically be effective for quality improvement, the results of empiric studies have been mixed, even if multiple reminders are sent or the reminders are delivered electronically. ${ }^{15-28}$ However, point-of-care evidence reminders such as the ones we used have been reported to have a more substantial effect when coupled with an alerting element that "pushes" the clinician to change prescribing. ${ }^{26-28}$ For example, we were previously able to significantly improve bisphosphonate use among patients with osteoporosis by use of faxed opinion leader statements that were identical in format to those used in this study. However, that study included patient activation to "push" the evidence reminder (i.e., study nurses recommended to patients in the intervention group that they see their physician to discuss osteoporosis therapy). ${ }^{28}$ This mirrors the results of our earlier study which showed that activation of patients via decision aids can induce short-term improvements in the quality of care for patients with atrial fibrillation..$^{29}$

\section{Strengths and limitations}

Although we conducted a cluster randomized trial with blinded outcome ascertainment, there are some limitations to our study. First and most importantly, we underestimated the magnitude of the improvement that would occur in our control patients. Although the diagnosis of coronary disease at cardiac catheterization is a "teachable moment," which would be expected to precipitate changes in management, the greater than expected improvements in our controls may be partly explained by the fact that our control patients were not "usual care" controls. The primary care physicians of our control patients received a faxed coronary artery diagram, which likely served as a point-of-care reminder even in the control arm. Indeed, the $82 \%$ use of statins and $55 \%$ use of triple therapy in our control group 6 months after catheterization were substantially higher than the $63 \%$ and $29 \%$ observed in an analysis of patients who met the ESP-CAD criteria but who were enrolled in the APPROACH study in the 2 years before launch of our study. ${ }^{30}$

Second, we do not know whether the intervention patients' physicians saw the intervention materials nor their opinions of the content. Third, because we recorded medication use at 6 months by patient self-report (with cross-referencing with pharmacy records), we do not know the extent to which patient noncompliance may have led us to underestimate the efficacy of our intervention on the prescribing patterns of 
their physicians. Fourth, our study was not designed to detect differences in health outcomes; however, the secondaryprevention therapies that we measured have been shown to be efficacious in reducing cardiovascular mortality.

Although our interventions might be considered "low-tech" given the advent of electronic health records with embedded computerized decision support, it should be noted that few primary care physicians currently use electronic records with such advanced functionality. ${ }^{31}$ Prior studies ${ }^{32}$ have shown that the modality of delivery of evidence prompts (computer-based v. paper-based) does not influence effectiveness. Although some may argue that unimodal interventions such as ours are too weak to influence practice, it is worth noting that several studies ${ }^{26-28}$ have shown that relatively minimalist interventions can substantially affect practice and quality of care. Indeed, multifaceted interventions are not necessarily more effective than single interventions, ${ }^{33}$ and to feasibly improve quality for common conditions dealt with largely in the outpatient setting (such as coronary artery disease), it is necessary to develop and evaluate minimalist interventions.

Finally, unlike prior studies of point-of-care reminders endorsed by specialists or local opinion leaders ${ }^{15-28}$ our inclusion of 2 intervention arms in the ESP-CAD study permits us to disentangle the effect of local opinion leaders from that of the evidence statements (i.e., to separate the messenger from the message). We observed a greater effect of evidence statements that were endorsed by local opinion leaders compared with the anonymous (but otherwise identical) unsigned statements, although the $6 \%$ absolute difference was not statistically significant.

\section{Conclusion}

Although evidence summaries endorsed by local opinion leaders led to improvements in the quality of statin management, which may have been clinically important $(10 \%$ absolute difference), our study was underpowered to detect if this difference was statistically significant, given the higher than anticipated improvements in the control arm. Regardless, our results suggest that faxed evidence reminders to primary care physicians are insufficient on their own to optimize the quality of care for patients with chronic coronary artery disease in the outpatient setting. Some form of stimulus in addition to point-of-care reminders (e.g., patient activation, ${ }^{28,29}$ involvement of allied health professionals ${ }^{34}$ ) appears to be needed to optimize the care, and hence outcomes, for patients.

\section{This article has been peer reviewed.}

Competing interests: Ross Tsuyuki has received research grants from Merck Frosst, Astra-Zeneca and Apotex. He has received speaker fees from Sanofi-Aventis, Novartis, Apotex, Merck Frosst and Pfizer. None declared for Finlay McAlister, Miriam Fradette, Sumit Majumdar, Randall Williams, Michelle Graham, James McMeekin, William Ghali, Merril Knudtson and Jeremy Grimshaw.

Contributors: Finlay McAlister conceived and designed the study and drafted the manuscript. Miriam Fradette, Randall Williams, Michelle Graham, James McMeekin and Merril Knudtson contributed to the acquisition of data. All authors gave input regarding study concept and design, interpreted data, provided comments on drafts, revised the manuscript critically for important intellectual content, and approved the final version of the manuscript submitted for publication.
Acknowledgements: The authors thank the opinion leaders (Drs. Paul Greenwood, Zaheer Lakhani, and TK Lee in the Edmonton region; Drs. Todd Anderson, James Cohen, Patrick Ma, Don Meldrum and James Stone in the Calgary region), the primary care practices and the physicians, nurses and technicians in the cardiac catheterization laboratories at the Foothills Medical Centre in Calgary, the Royal Alexandra Hospital and University of Alberta Hospital in Edmonton for their support in data collection for APPROACH. The authors also thank the ESP-CAD study nurses (Kathleen Hempstock, Kate Clarke, Carol Cysouw, Laura Haan, Maxine Mulligan and Beverley Madden), the staff of the EPICORE Centre involved with this study (Ruth Dupuit, Marilou Hervas-Malo, Sipi Garg, Betty Larson, Leah Foley, Lily Yushko and Paula Priest), and the APPROACH staff (in particular Diane Galbraith, Prince Michael and Chris Hopp) and Dr. Colleen Norris.

Finlay McAlister and Sumit Majumdar hold career salary support from the Alberta Heritage Foundation for Medical Research (AHFMR). Finlay McAlister and Ross Tsuyuki are supported by the Merck Frosst/Aventis Chair in Patient Health Management at the University of Alberta. William Ghali is supported by the AHFMR and a Canada Research Chair.

Funding: APPROACH was initially funded with a grant from the W. Garfield Weston Foundation. The ongoing operation of APPROACH has been made possible by contributions from the Provincial Wide Services Committee of Alberta Health and Wellness as well as the Libin Cardiovascular Institute and Mazankowski Heart Institute and the following industry sponsors: Merck Frosst Canada Inc., Roche Canada, Eli Lilly Canada Inc., Bristol-Myers Squibb, Philips Medical Systems Canada, Searle Pharmaceuticals, Boston Scientific Ltd and Cordis.

The ESP-CAD Trial was funded by 3 peer-reviewed grants (Alberta Heritage Foundation for Medical Research, the Heart and Stroke Foundation of Canada and Pfizer Canada Inc). None of the funding organizations had a role in the conception or design, conduct, analysis, interpretation, or reporting of the study, and none had access to the data. None of the investigators or local opinion leaders received any financial compensation.

\section{REFERENCES}

1. McAlister FA, Fradette M, Graham M, et al. A randomized trial to assess the impact of opinion leader endorsed evidence summaries on the use of secondary prevention strategies in patients with coronary artery disease: The ESP-CAD trial protocol (NCT00175240). Implement Sci 2006;1:11.

2. Bhatt DL, Steg PG, Ohman EM, et al. International prevalence, recognition, and treatment of cardiovascular risk factors in outpatients with atherothrombosis. JAMA 2006;295:180-9.

3. EUROASPIRE I and II Group. Clinical reality of coronary prevention guidelines: a comparison of EUROASPIRE I and II in nine countries. Lancet 2001;357:995-1001.

4. Pilote L, Beck CA, Karp I, et al. Secondary prevention after acute myocardial infarction in four Canadian provinces, 1997-2000. Can J Cardiol 2004;2:61-7.

5. Newby LK, Allen LaPointe NM, Chen AY, et al. Long-term adherence to evidence-based secondary prevention therapies in coronary artery disease. Circulation 2006;113:203-12.

6. Ford ES, Ajani UA, Croft JB, et al. Explaining the decrease in U.S. deaths from coronary disease, 1980-2000. N Engl J Med 2007;356:2388-98.

7. Majumdar SR, McAlister FA, Furberg CD. From publication to practice in chronic cardiovascular disease - the long and winding road. J Am Coll Cardiol 2004;43:1738-42.

8. Majumdar SR, Tsuyuki RT, McAlister FA. Impact of opinion leader endorsed evidence summaries on quality of prescribing for patients with cardiovascular disease: randomized controlled trial (ISRCTN26365328). Am Heart J 2007;153:22-9.

9. Ghali WA, Knudtson ML; for the Alberta Provincial Project for Outcome Assessment in Coronary Heart Disease (APPROACH) Investigators. Overview of the Alberta Provincial Project for Outcome Assessment in Coronary Heart Disease. Can J Cardiol 2000;16:1225-30.

10. McPherson R, Frohlich J, Fodor G, et al. Canadian Cardiovascular Society position statement - recommendations for the diagnosis and treatment of dyslipidemia and prevention of cardiovascular disease. Can J Cardiol 2006;22:913-27.

11. Diggle PJ, Liang KY, Zeger SL. Analysis of longitudinal data. New York (NY): Oxford University Press; 1996

12. Sacks H, Chalmers TC, Smith H. Randomized versus historical controls for clinical trials. Am J Med 1982;72:233-40.

13. Auerbach AD, Landefeld CS, Shojania JG. The tension between needing to improve care and knowing how to do it. N Engl J Med 2007;357:608-13.

14. Josan K, Majumdar SR, McAlister FA. The efficacy and safety of intensive statin therapy: a meta-analysis of randomized trials. CMAJ 2008;178:576-84.

15. Grimshaw JM, Shirran L, Thomas R, et al. Changing provider behavior. An overview of systematic reviews of interventions. Med Care 2001;39:II2-45.

16. Guadagnoli E, Normand SLT, DiSalvo TG, et al. Effects of treatment recommendations and specialist intervention on care provided by primary care physicians to patients with myocardial infarction or heart failure. Am J Med 2004;117:371-9.

17. Derose SF, Dudl JR, Benson VM, et al. Point of service reminders for prescribing cardiovascular medications. Am J Manag Care 2005;11:298-304. 


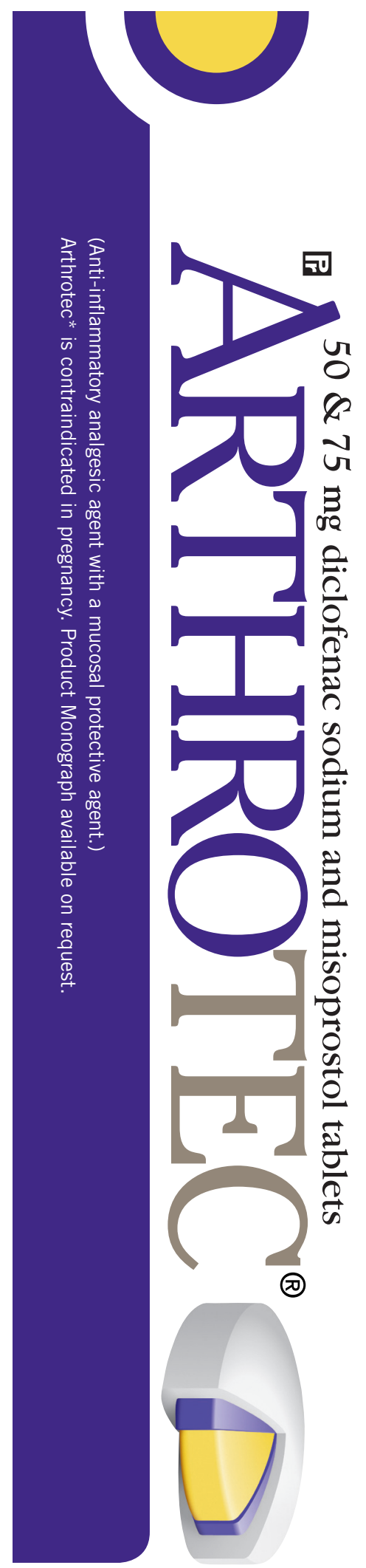

\section{RESEARCH}

18. Feder G, Griffiths C, Eldridge S, et al. Effect of postal prompts to patients and general practitioners on the quality of primary care after a coronary event (POST): randomised controlled trial. BMJ 1999;318:1522-6.

19. Eccles M, McColl E, Steen N, et al. Effect of computerized evidence based guidelines on management of asthma and angina in adults in primary care: cluster randomized controlled trial. BMJ 2002;325:941-7.

20. Tierney WM, Overhage JM, Murray MD, et al. Effects of computerized guidelines for managing heart disease in primary care. J Gen Intern Med 2003;18:967-76.

21. Demakis JG, Beauchamp C, Cull WL, et al. Improving residents' compliance with standards of ambulatory care. Results from the VA Cooperative Study on Computerized Reminders. JAMA 2000;284:1411-6.

22. Lester WT, Grant RW, Barnett GO, et al. Randomized controlled trial of an informatics-based intervention to increase statin prescription for secondary prevention of coronary disease. J Gen Intern Med 2006;21:22-9.

23. Sequist TD, Gandhi TK, Karson AS, et al. A randomized trial of electronic clinical reminders to improve quality of care for diabetes and coronary artery disease. $J \mathrm{Am}$ Med Inform Assoc 2005;12:431-7.

24. van Wyk JT, van Wijk MAM, Sturkenboom MCJM, et al. Electronic alerts versus on-demand decision support to improve dyslipidemia treatment. A cluster randomized controlled trial. Circulation 2008;117:371-8.

25. Sales A, Helfrich C, Ho PM, et al. Implementing electronic clinical reminders for lipid management in patients with ischemic heart disease in the Veterans Health Administration: QUERI Series. Implement Sci 2008;3:28.

26. Thomas RE, Croal BL, Ramsay C, et al. Effect of enhanced feedback and brief educational reminder messages on laboratory test requesting in primary care: a cluster randomised trial. Lancet 2006;367:1990-6.

27. Eccles M, Steen N, Grimshaw J, et al. Effect of audit and feedback, and reminder messages on primary-care radiology referrals: a randomised trial. Lancet 2001;357:1406-9.

28. Majumdar SR, Johnson JA, McAlister FA, et al. Multifaceted intervention to improve diagnosis and treatment of osteoporosis in patients with recent wrist fracture: a randomized controlled trial. CMAJ 2008;178:569-75.

29. McAlister FA, Man-Son-Hing M, Straus SE, et al.; DAAFI Investigators. The impact of patient decision aids on care in patients with atrial fibrillation: a cluster randomized trial. CMAJ 2005; 173:496-501.

30. McAlister FA, Oreopoulos A, Norris CM, et al; for the Alberta Provincial Project for Outcome Assessment in Coronary Heart Disease (APPROACH) Investigators. Exploring the treatment-risk paradox in coronary disease. Arch Intern Med 2007; 167:1019-25.

31. DesRoches CM, Campbell EG, Sowmya RR, et al. Electronic health records in ambulatory care: a national survey of physicians. N Engl J Med 2008;359:50-60.

32. Balas EA, Weingarten $\mathrm{S}$, Garb CT, et al. Improving preventive care by prompting physicians. Arch Intern Med 2000;160:301-8.

33. Grimshaw JM, Thomas RE, MacLennan G, et al. Effectiveness and efficiency of guideline dissemination and implementation strategies. Health Technol Asses. 2004:8:iii-iv, 1-72.

34. McLean DL, McAlister FA, Johnson JA, et al. A randomized trial of the effect of community pharmacist and nurse care on improving blood pressure management in patients with diabetes: SCRIP-HTN (NCT00374270). Arch Intern Med 2008;168:2355-61.

Correspondence to: Dr. Finlay A. McAlister, Division of General Internal Medicine, 2F1.21 Walter Mackenzie Health Sciences Centre, University of Alberta Hospital, 8440-112th St., Edmonton AB T6G 2B7; fax 780 407-3132; finlay.mcalister@ualberta.ca

\section{Reprints}

\section{Bulk reprints of $C M A J$ articles are available in minimum quantities of $\mathbf{5 0}$}

For information or orders:

Reprint Coordinator

tel 800 663-7336 x2110, fax 613 565-7704

janis.murrey@cma.ca 